\title{
Analysis of simple inventory control systems with execution errors: Economic impact under correction opportunities
}

\author{
Esma S. Gel ${ }^{\mathrm{a}, *}$, Nesim Erkip ${ }^{\mathrm{b}}$, Anoop Thulaseedas ${ }^{\mathrm{a}}$ \\ a Arizona State University, School of Computing, Informatics, and Decision Systems Engineering, P.0. Box 878809, Tempe, AZ 85287-8809, USA \\ ${ }^{\mathrm{b}}$ Bilkent University, Department of Industrial Engineering, Ankara, 06800, Turkey
}

\section{A R T I C L E I N F O}

\section{Article history:}

Received 13 July 2007

Accepted 16 January 2010

Available online 29 January 2010

Keywords:

Inventory control

Simulation

RFID

Inventory record inaccuracies

Execution errors

\begin{abstract}
A B S T R A C T
Motivated by recent empirical evidence, we study the economic impact of inventory record inaccuracies that arise due to execution errors. We model a set of probable events regarding the erroneous registering of sales at each demand arrival. We define correction opportunities that can be used to (at least partially) correct inventory records. We analyze a simple inventory control model with execution errors and correction opportunities, and demonstrate that decisions that consider the existence of recording errors and the mechanisms with which they are corrected can be quite complicated and exhibit complex tradeoffs. To evaluate the economic impact of inventory record inaccuracies, we use a simulation model of a $(Q, r)$ inventory control system and evaluate suboptimalities in cost and customer service that arise as a result of untimely triggering of orders due to inventory record inaccuracies. We show that the economic impact of inventory record inaccuracies can be significant, particularly in systems with small order sizes and low reorder levels.
\end{abstract}

(c) 2010 Elsevier B.V. All rights reserved.

\section{Introduction}

Global pressures for high customer service levels have placed a strong emphasis on the control of material flow in today's production and retail environments. Companies are constantly in search of efficient systems and procedures to manage the levels of various types of stocks in their systems. For that purpose, many of them have made substantial investments in Information Technology (IT) to automate various functions, such as supply chain operations. It is estimated that US retailers spend close to $\$ 30$ billion annually to make supply chain decisions like merchandise tracking, automating transactions, and inventory level optimization (Raman et al., 2001).

IT system implementations in supply chains have made abundant data available, which has motivated many possibilities of system improvement through the use of that data. The research community has been analyzing the various types of savings inherent in sharing and analysis of these data to show the importance of IT for effective supply chain management. One issue that has been somewhat neglected, however, is the effect of inaccurate data in decision making. Strategies that have been shown to be optimal or near-optimal assuming availability of perfect information may not behave so if implemented in a system with sensors that are only able to provide inaccurate data.

\footnotetext{
* Corresponding author. Tel.: +1 480965 2906; fax: +1 4809658692.

E-mail address: esma.gel@asu.edu (E.S. Gel).
}

Monitoring and replenishment of stocks is becoming an automated function in many companies. These systems use advanced database structures as well as a set of sensors, such as barcode readers and Radio Frequency Identification (RFID) tags, to track the number of products in the system and place timely orders to the supplier(s) according to the inventory control policy in place. Management infrastructures, such as ERP systems, control almost all functions regarding finance, marketing, logistics, and manufacturing of goods based on this data. Optimality or effectiveness of decision policies can only be valid under the conditions that data is perfect; inaccuracies in data can often result in suboptimal performance, sometimes without the apparent knowledge of decision makers. Hence, accuracy of data regarding the location and quantity of goods is critical for the profitability of a company.

The introduction of IT systems, which replaced manual inventory record keeping, held a lot of promise for the elimination of inventory inaccuracies. Today, these IT systems as well as the sensors that collect the data are improving (and requiring additional investments) everyday; however, errors in inventory records still exist. In fact, discrepancies between inventory records in information systems and physical inventory are quite common. The existence and extent of such discrepancies, which we refer to as inventory record inaccuracies, have been documented in the literature by several researchers as well as industry reports. Raman et al. (2001) studied the inventory records of two leading retailers and found that inventory records of almost $65 \%$ of the SKU's were inaccurate. Furthermore, the magnitude of 
errors on average was found to be $35 \%$ of the target stock levels. Similar observations were made by Kang and Gershwin (2005), based on their experiences with several retailers considering the adoption of an RFID-based IT technology.

Inventory audit reports from various environments ranging from hospital pharmacies to the grocery industry echo the prevalence of inventory record inaccuracies. These reports document the excess inventories that companies carry as a result of inventory record inaccuracies, and the significant economic impact. On one of these audits, for example, the Office of Inspector General estimated that at any given time the value of Veteran's Health Administration-wide excess inventory was worth $\$ 64.1$ million, which was 61.8 percent of the $\$ 103.8$ million total inventory. Of the $\$ 64.1$ million in excess inventory, at least $\$ 10.8$ million was inventory for which there was no demand. The report ${ }^{1}$ also draws attention to the fact that the excess inventories occurred because of inadequate or erroneous monitoring of stock levels.

In general, the actions that companies take to respond to inventory record inaccuracy can be grouped in three categories: (1) prevention, (2) correction, and (3) integration (DeHoratius et al., 2008). Prevention strategies aim to reduce or eliminate the root causes of inaccuracies through improvements in education of workforce, product and shipment labeling, shelving and storage of items, foolproofing of procedures, information technology, and product tagging, etc. While these actions will obviously reduce inventory record inaccuracies, factors such as labor turnover, human error, sensory and tracking equipment failures imply that it is extremely hard, if not impossible, to eliminate inventory record inaccuracies. For this reason, most companies resort to correction of inventory records. These mainly consist of auditing policies to identify and correct record discrepancies. While annual physical inventories are performed at the end of every year by all companies for accounting purposes, many companies also perform cycle counting, which is the practice of periodically counting all or a fraction of the on-hand inventory. The third category, integration, involves the use of inventory management strategies that explicitly consider the existence of inventory record inaccuracies and incorporate this into the decision making process. These approaches include the use of appropriate auditing cycles (e.g., Iglehart and Morey, 1972; Morey, 1985; Morey and Dittman, 1986; Kok and Shang, 2007), compensation methods that take stochastic behavior of stock loss into account (e.g., Kang and Gershwin, 2005), modified replenishment policies (e.g., Lee and Özer, 2007; Atalı et al., 2009), and policies based on the Bayesian inventory record for replenishment and audit triggering (DeHoratius et al., 2008).

Regardless of the mix of actions companies choose to take to tackle record inaccuracies, costs for prevention, correction and integration can be significant. Therefore, it is important to determine the "true" economic impact of inaccuracies due to various major causes so that costs to address these causes can be justified. In particular, the impact of inaccuracies due to different causes (which we detail in Section 2) should be accounted for separately, since actions to address different causes may be quite different. Consider for example, theft and execution errors at the cash registers: while increasing security may be a way to address inventory shrinkage due to theft, it would do little to reduce inaccuracies due to execution errors. To justify the costs for actions to minimize execution errors (such as those for training, labeling, shelving) one needs to determine the economic impact

\footnotetext{
1 Available at http://www.va.gov/oig/52/reports/1999/9R8-E04-052.pdf as of
} $12 / 31 / 2009$ of various execution errors that cause over- and under-registering of demand.

To come up with reliable estimates of economic impact, it is necessary to use a model that represents reality as closely as possible. In particular, one should consider various types of naturally occurring events that "help" companies keep the inaccuracies under control on a daily basis. One such event, for example, is the case in which a customer brings to the cash register an item that appears to be stocked out. This event triggers at least a partial correction of the inventory records and can be used to trigger other types of record correction mechanisms. As we outline below, it is possible to come up with other such events, which we refer to as "correction opportunities". Modern IT systems are capable of collecting, storing and processing massive amounts of data, which means that it is also possible to take corrective action using correction opportunities on a real-time basis. To our knowledge, there is very limited work on how to take advantage of correction opportunities in retail/production environments. In addition to providing more accurate estimates of economic impact, consideration and modeling of these events are important building blocks that could lead to the development of self-healing systems.

In this paper, we focus on modeling execution errors and various events that indicate record inaccuracies in the system to provide reliable estimates of the economic impact of execution errors. Our approach isolates execution errors and allows for positive and negative record inaccuracies, as opposed to studies that only consider positive record inaccuracies due to theft and inventory shrinkage. We pay close attention to the stochastic modeling of errors as they occur at demand arrival epochs, which allows us to tie the economic impact to the scale of errors and to generalize our results to various retail environments with possibly different levels of execution errors. To show the complexity of the problem, we start our discussion with a simple model with execution errors and correction opportunities to demonstrate the impact of over-registering demand and how the optimal ordering quantity changes as a function of the error level and correction probability. We then present parallel results using a simulation model of a single item inventory system managed by a continuous review inventory control policy. We compare the expected total cost (sum of holding, ordering and lost sales costs) of the optimal $(Q, r)$ policy and fraction of lost sales with and without execution errors. We provide some results on using indicator events as opportunities to completely or partially correct inventory records. Finally, we summarize a set of managerial insights that practitioners can use to assess the economic impact of record inaccuracies.

In Section 2, we present an overview of the prior work on the subject as well as a categorization of common inventory record inaccuracies. A statement of contribution in contrast to other work in the literature is also provided. Section 3.1 provides a detailed description of the inventory control system we consider, as well as a definition of correction opportunities. In Section 3.2 we present a simplistic analytical inventory control model as well as several managerial insights derived from its analysis. Section 4.1 includes an outline of the simulation model and experimental design. In the remaining subsections we discuss our findings from the simulation experiments. In Section 5 we provide some managerial insights. We finally present conclusions and directions for future work in Section 6.

\section{Literature review}

Inventory inaccuracies have been known to exist, which is the reason why most companies have adopted a policy of conducting 
cycle counts at least once a year. While almost all research on inventory policies assumes perfect information on inventory levels, there has been some early work on the determination of the effective frequency of inventory audits. Iglehart and Morey (1972) considered a reorder point policy, and found the optimal combination of additional safety stock and frequency of cycle counts that minimizes the sum of holding and inspection costs. Morey (1985) and Morey and Dittman (1986) consider similar issues in the determination of an effective frequency of inventory audits and safety stocks. Fleisch and Tellkamp (2005) simulate a three-echelon retail supply chain to study the effect of inventory inaccuracies arising due to low process quality, theft and items becoming unsaleable, and show that elimination of inventory inaccuracy can reduce the supply chain costs. In a more recent paper, Gümrükçü et al. (2008) present a simulation model of a two-echelon inventory system consisting of a retailer, a distribution center, and a supplier, and examine an extensive set of cycle counting configurations to observe the tradeoff between fill rates, accuracy and system costs for a multi-item system. Their results also point to the importance of correct application of cycle counting.

The question of when and for which item to conduct an inventory audit has received considerable attention in earlier work. Cantwell (1985), Edelman (1984), Reddock (1984), and Neely (1987) considered classification approaches to differentiate between the various SKUs of a company; Buck and Sadowski (1983) and Martin and Goodrich (1987) considered sampling from the various SKUs. Bernard (1985) and Graff (1987) considered managerial steps to make inventory audits more effective, emphasizing that cycle counts alone are inadequate to control or improve accuracy. Young and Nie (1992) used a simulation study to assess the effect of cycle counting strategies on stock-out risk under two inventory order policies.

Recently, there has been some work that considers similar issues within the context of optimal inventory control. Kok and Shang (2007) study the problem of minimizing total inspection and inventory costs and show that the inspection-adjusted basestock policy is optimal for a single-product, single-stage inventory system. Under this policy, an inspection is made if the recorded inventory is less than a threshold level. Upon verification of the inventory level, a base-stock policy is followed. In a similar spirit, Atalı et al. (2009) propose inventory policies that account for inventory inaccuracies. Both studies compare the performance of the proposed policies to RFID-enabled inventory systems with complete inventory visibility to provide an understanding for the true value of RFID in inventory management. Bensoussan and Sethi (2005) consider a partially-observed inventory system where the demand is not observed and inventory level is confirmed when it reaches zero, and develop optimal or approximately optimal feedback policies to determine the replenishment orders that minimize the total discounted costs over an infinite horizon.

With reductions in the cost of RFID tags and improvements in data mining techniques, companies have intensified their efforts in implementing RFID. The visibility that RFID can bring to the supply chain has been cited to have significant potential to save labor costs, improve supply chain coordination, reduce inventory, and increase product availability and customer service. However, most of the claims on the benefits of RFID have not been carefully substantiated using quantitative, realistic models. Lee and Özer (2007) argue that there currently exists a "credibility gap" of the value of RFID, since most assessments of the value of RFID fail to disclose how the estimated values of RFID were determined and how those values can be realized by companies implementing RFID. The authors draw attention to this gap in the literature, and identify this as an area that needs to be strengthened through further modeling.
One particular type of inaccuracy that has received considerable attention concerns the overestimation of the actual stock; that is, the actual stock level is lower than that indicated by the IT system. The term shrinkage is the retail parlance for such discrepancies. Beck and Chapman (2003) divide inventory shrinkage into two categories: malicious and non-malicious shrinkage. Malicious shrinkage refers to incidences of internal or external theft, unauthorized consumption, and fraud, whereas non-malicious shrinkage refers to incidences of unintentional errors by employees, inaccessibility, obsolescence, or spoilage. Inventory shrinkage (a.k.a. stock loss) happening without the knowledge of store personnel creates inventory record inaccuracy. These discrepancies between the actual stock levels and stock levels indicated in the inventory records mean that untimely orders are triggered and on the average, more stockouts than intended are incurred.

Kang and Gershwin (2005) consider the impact of inventory shrinkage in terms of the average number of stockouts to justify investment in an automatic production identification technology developed at the Auto-ID Center at MIT. In particular, the authors use the relationship between the stock level at the beginning of a period, inbound shipments, number of sales, and stock loss incidents during a period to simulate a system with unknown stock loss. The stock loss incidents were generated from a Poisson distribution, independently from demand occurrences. The analysis indicates that inventory shrinkage can lead to increased stockouts with significant revenue losses in systems with lost sales. Kang and Gershwin (2005) point to the interesting managerial insight that the effect of items lost to shoplifters, for example, is in fact much higher than the direct loss incurred by the retailer. The inaccuracies as a result of stock loss lead to a chain reaction of untimely replenishments and increased stockouts, which means that the actual cost to the retailer is much higher.

Execution errors consist of another major source of errors contributing to inventory record inaccuracies. These errors range from incorrect accounting of inbound shipments due to mistakes in product identification or labeling to execution errors at cash registers. Consider, for example, a retail outlet that has invested in a sensor network to manage its inventory. The sales information at the Point-of-Sales (POS) locations goes directly into the database that maintains the inventory records. In addition to updating the inventory data to register the sales, the IT system automatically makes replenishment decisions based on the inventory level in the database. Customers come into the store, pick up the items they need, and bring them to the checkout counter. The store employee at the checkout counter scans the barcode of the items to register sales, which updates the inventory levels in the database.

Consider a customer buying ten packets of noodles-five packets of one flavor and five packets of another. Nowadays, it is common for an item to come in different variants with the same cost, e.g., packets of noodles with different flavors that cost the same. To facilitate faster processing, the sales person at the checkout counter may scan the barcode of one of the packets to register a sale of ten packets. This technique works well when it comes to saving time; however, the procedure causes inaccurate data to be recorded into the IT database. After this transaction, the IT system will show a ten-packet reduction in the stock of the item whose barcode was scanned, and no change for the other item(s). It is possible to list similar scenarios happening during product returns or exchanges. Note that these errors are mainly due to poor execution or incorrect use of sensors, such as barcode scanners. Execution errors differ from stock loss in the sense that the discrepancies can be positive or negative; that is, the actual stock level may be lower or higher than the stock level indicated 
by the inventory records maintained by the IT system. Just like inventory shrinkage, execution errors lead to untimely replenishments and increased stockouts. In addition, these errors will lead to increased errors in demand forecasts. Hence, the effect of execution errors can even propagate up the supply chain and contribute to the bullwhip effect through the errors introduced into the demand forecasts.

A number of studies consider different environments where execution errors of a certain kind play a role in the inventory system that is defined as a kind of supply uncertainty. Rekik et al. (2008a) considers the misplacement of inventory situation almost similar to unreliable supplier problem. They optimize the known situation, as well as using RFID assume perfect system to make comparisons. Sahin et al. (2008) and Sahin and Dallery (2009) both consider the effect of inventory inaccuracy for a wholesaler. Sahin et al. (2008) considers a system were execution errors result in a discrepancy between the physical inventory and information system, the discrepancy represented by an additive distribution. Sahin and Dallery (2009), on the other hand, considers a multiplicative representation for the uncertain quantity. The same work includes a model that represents an error in the physical inventory, as well. Rekik et al. (2009) considers a multiperiod problem where the records are inaccurate as a result of theft. Similar models are constructed to show possible benefits of a more capable technology.

Another group of studies considers a similar approach to the above for the supply chain, where supply chain is made up of a manufacturer and a retailer. Rekik et al. (2007) and Rekik et al. (2008b) consider execution errors due to incorrect accounting of inbound shipments and misplacement of items. Under a singleperiod setting, the authors analyze the effectiveness of different technologies on the performance of the supply chain as a whole; the supply chain defined as decentralized or centralized. In Rekik et al. (2007) they also consider coordinating contract structures. Note that, in all of the above studies, as a result of their assumptions, execution errors lead to a total amount available for sale that is less than that indicated by the IT system records.

This paper presents descriptive models to understand the economic impact of inventory record inaccuracies so that a framework for prescriptive studies can be established. In particular, we develop a detailed model of an inventory control environment operating under a continuous review policy. In contrast to studies that evaluate the effects of inventory loss, such as Kang and Gershwin (2005), we consider execution errors that result in both over-recording and under-recording of demand. In our simulation studies, we consider costs over a finite horizon, as opposed to other studies that assume an infinite horizon or a single-period.

In addition to modeling execution errors that occur at demand arrivals, we model correction epochs (such as cycle counts and incidences that signal the existence of errors) and likely actions to fully or partially correct records. We define two events that signal the existence of errors and refer to them as "correction opportunities." In this respect, one of the closest studies to ours is Mosconi et al. (2004), which considers the impact of inventory inspection policies (such as zero-balance walks) on the expected level of inventory record inaccuracy in their working paper. Zerobalance walks can be considered as a special type of correction opportunity. In this respect, we see the work of Mosconi et al. (2004) as complementing to ours. Another study in this vein is Gümrükçü et al. (2008), which acknowledges the idea of correction opportunities by extending the classical cycle counts to opportunity counts. Their study, however, is different than ours, since they use the correction opportunities as possible signals for cycle counts, whereas we inquire the possibility and potential of making use of these events more directly.
Our work is the one of the first studies that considers the comprehensive set of correction opportunities (to the extent enabled by information technologies used) that occur naturally in real-life systems. Hence, our results address the need for more realistic models to assess the true impact of inaccuracies Lee and Özer (2007).

\section{Analysis}

\subsection{Description of the inventory control system}

We consider the management of stock levels of a single item with high demand and high service level requirements. An example to such systems can be retail environments. We assume that stock levels are controlled using a continuous review policy. In particular, we assume that an optimal or near-optimal $(Q, r)$ policy, under which an order of $Q$ units is triggered when the IT system observes an inventory position of $r$ or less, is used. We assume that demand arrivals are random and occur independently of each other. We study two cases: (i) each customer demanding a single unit, and (ii) each customer demanding a random number of units. We assume inventory stockouts result in lost sales. We consider inventory holding costs, order setup costs, lost sales costs and variable purchasing costs. Inventory holding costs are incurred on the basis of actual on-hand inventory, rather than that indicated by the inventory records.

We focus on execution errors that occur at demand arrivals. In particular, we are interested in erroneous registering of the number of units sold. These errors result in either an "overregistering" of demand (i.e., actual stock higher than that indicated by inventory records) or an "under-registering" of demand (i.e., actual stock less than that indicated by inventory records). Since the recorded inventory levels may be different than those actually in stock, a customer demand may still be lost when the IT system shows positive inventory, or a customer demand may be satisfied when the IT system shows an inventory of zero. Both of these cases are treated as Correction Opportunities ( $\mathrm{CO}$ ) that can be utilized to improve system performance:

- CO-1: The store experiences a stockout (i.e., a customer indicates that the item cannot be found on the shelf) while the inventory record shows positive inventory,

- CO-2: A customer (or store employee) finds an item and brings it to the cash register when the inventory record for that item is zero.

In the first correction opportunity, we assume that the stockout incident can be detected by feedback from customers. Some retail outlets also use an inspection policy called zero-balance walks; store employees walk the aisles to identify items that have stocked out. Mosconi et al. (2004) study the impact of different inspection procedures, one of them being zero-balance walks, on the expected level of inventory record inaccuracy.

$\mathrm{CO}-1$ is an effective correction opportunity, since the inventory record can be completely corrected upon a $\mathrm{CO}-1$ incident. On the other hand, only a partial correction is possible with CO-2; the inventory level right before the sale (which is zero) can be adjusted to account for the number of items brought by the customer to the point of sale. It is useful to note here that there is, indeed, a way to ensure that a $\mathrm{CO}-2$ incident results in a complete correction by enabling a counting of the remaining products in the shelf. We do not consider this more extensive version of $\mathrm{CO}-2$ in our studies. 


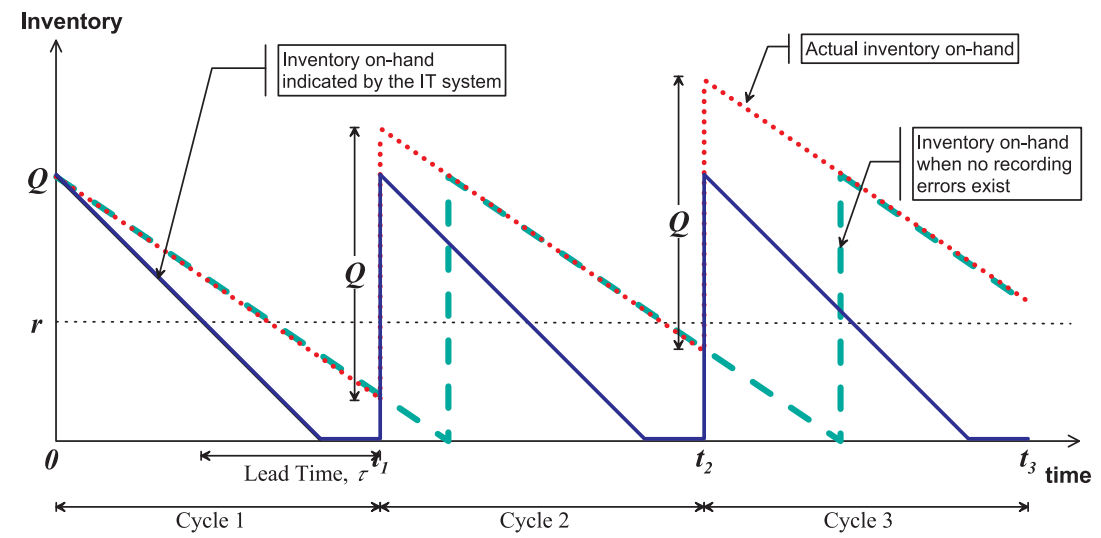

Fig. 1. Level of inventory over time in a system that over-registers demand.

To the best of our knowledge, this is the first study that considers correction opportunities that occur naturally in the system. There are two main reasons for considering these natural correction opportunities: (i) Models that incorporate these natural correction opportunities reflect the performance of the inventory system correctly, as happens in real systems. (ii) The existence of correction opportunities is important and can be made instrumental in reduction of errors. Once a correction opportunity is observed, the system controller can update the IT system database to reflect this observation. Note that such correction opportunities, in contrast to other correction mechanisms like cycle counts, usually involve significantly less cost and worker effort.

\subsection{Insights on the impact of execution errors}

To develop some fundamental insights on the impact of execution errors, let's consider a simple model with deterministic demand. Let $Q$ and $r$ denote the order quantity and the reorder level, respectively. Note that $r=\tau D$, where $\tau$ denotes the lead time and $D$ denotes the constant demand rate (items per unit time). ${ }^{2}$

Let's consider the case of over-registering of demand. Suppose that each unit of demand is recorded as $1+p$ units of demand by the IT system, where $p$ is the "error level." Then, it is trivial to observe that the IT system will trigger a replenishment order earlier than a system with no record inaccuracies. Fig. 1 shows the inventory levels over time for this case. In the figure, the inventory profile shown by the solid line depicts the inventory on-hand indicated by the IT system, whereas the dotted inventory profile shows the actual inventory on-hand. For reference, the dashed inventory profile shows the corresponding inventory onhand for a system with perfect data. Note that since demand is deterministic, the order arrivals mark the beginning of cycles, which are indicated in the figure.

In Fig. 1, actual inventory accumulates at a constant rate in each cycle due to the early triggering of replenishment orders and the lost sales assumption. Without any mechanisms to reconcile inventory records and the actual stock levels, errors will accumulate over time, resulting in increases in inventory. A similar behavior is observed in Section 4.2, even in the case of symmetric errors in both directions of over-registration and under-registration of demand, due to the lost sales assumption.

\footnotetext{
${ }^{2}$ In general, if $\tau>Q / D$, the reorder point is given by $r=\tau D-Q\lfloor\tau /(Q / D)\rfloor$, where $\lfloor x\rfloor$ denotes the largest integer less than or equal to $x$. The derivations should be repeated for general $r$.
}

Note that such indefinite increases in inventory is not observed in real-life systems, since an inventory manager who observes such persistent behavior will implement corrective actions to reconcile the records. To model periodic reconciliation of records, suppose that every time an order arrives, there is an associated probability, $q$, such that the correction mechanism will be able to correct the recorded inventory level. For example, upon an order arrival, the company may perform a cycle count; a complete and perfect cycle count with no errors would correspond to a correction probability of one (i.e., $q=1$ ), whereas a partial cycle count may not be able to correctly determine the stock levels, resulting in a correction probability of less than one. We assume that this probability is stationary, although in real-life systems the correction probabilities may improve over time.

Let $K_{q}$ denote the cost of a correction mechanism with a correction probability of $q$. Note that $K_{q}$ includes costs to return or discard inventory, which may be necessary to bring the actual stock level to $Q$. Clearly, we expect that $K_{q}$ is an increasing function of $q$, since it is conceivable that more detailed audits would provide a better probability of correction, but at higher cost.

Appendix A provides a derivation of the expected total cost per unit time as well as several analytical properties that lead to the calculation of an optimal order size as a function of error rate, correction cost, and correction probability in addition to the usual parameters used in the economic order quantity calculation. From expression (A.7), we find that the optimal ordering quantity, $Q^{*}$, decreases as the error rate, $p$, increases. The reason for this behavior is that as the order size decreases, the number of possible correction epochs (i.e., order arrivals) increases. Hence, as error rate increases, it becomes more and more cost effective to utilize these possible correction epochs. Fig. 2 plots the optimal ordering quantity as a function of the error level, $p$. The following parameters were used for this case: $D=2,000$ units per year, $K=\$ 100$ per order, $c=\$ 2.00$ per unit, annual holding cost, $h=\$ 0.5$ per unit per year, and the reorder level, $r=80$. The correction probability is set at $q=0.20$. We plotted the optimal ordering quantity for three values of $K_{q}=75, K_{q}=200$, and $K_{q}=400$, as well as the economic order quantity, $\mathrm{EOQ}=\sqrt{2 K D / h}$ (which is equal to 895 units for this case).

From Fig. 2, we observe that the optimal ordering quantity is greater than EOQ for all values of $p$ if the correction cost, $K_{q}$ is sufficiently large. This is intuitive, since the system is trying to avoid excessive correction charges in addition to the setup costs by ordering large quantities. Similarly, when the correction cost, $K_{q}$ is low, the system orders in small quantities to take advantage of possible inventory record corrections at order arrivals, in addition to reducing inventory holding costs. In the $K_{q}=75$ and 


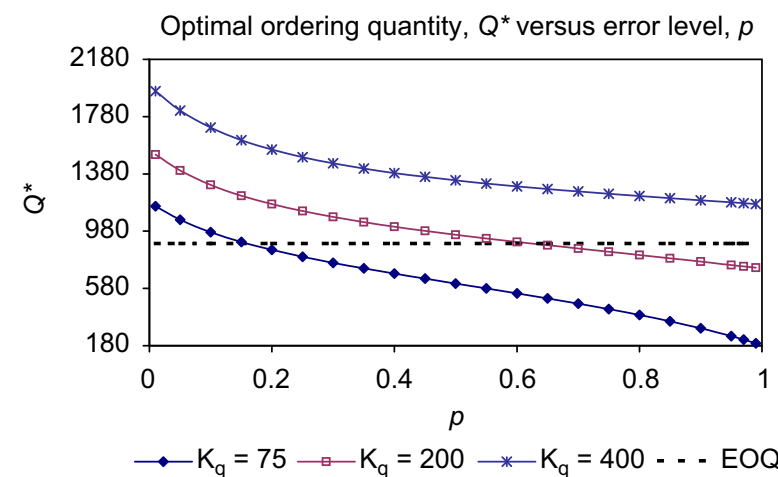

Fig. 2. Optimal ordering quantity versus error level, $p$.

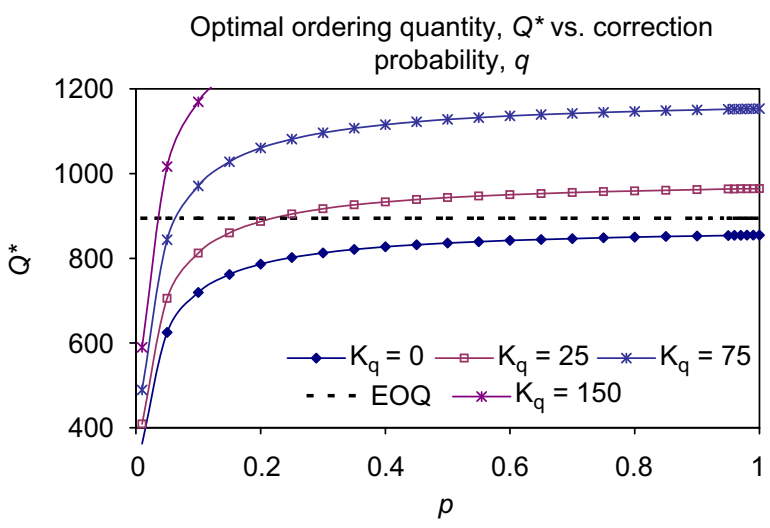

Fig. 3. Optimal ordering quantity versus correction probability, $q$.

$K_{q}=200$ cases, the system orders more than EOQ for small values of $p$, and orders less than EOQ for large values of $p$. This is also intuitive, since as $p$ gets large, the costs due to inventory record inaccuracies increase, and hence the system orders less to increase the frequency and take advantage of correction opportunities.

We also find that for constant error rate, $Q^{*}$ is an increasing function of $q$. Fig. 3 plots the optimal ordering quantity versus the correction probability, $q$ for three constant values of correction cost, $K_{q}=0, K_{q}=\$ 25, K_{q}=\$ 75$, and $K_{q}=\$ 150$. The same values for $D$, $K, c, h$ and $r$ are used with an error level of $p=0.05$. We see from the figure that when the fixed correction cost is zero (or very small), the optimal ordering quantity is less than the EOQ for all values of $q \leq 1$. However, for larger values of $K_{q}$, the optimal ordering quantities are higher than the EOQ except for very low values of correction probability, $q$. As the correction cost, $K_{q}$ increases, frequent orders become excessively expensive since these costs are incurred at order arrivals (just like the fixed setup costs). Hence, for sufficiently expensive corrections, the system prefers to incur inventory holding costs due to the build-up of inventory, rather than incurring setup and correction costs as a result of frequent ordering. When $K_{q}=0$ (or small), the optimal ordering quantity is small since although small order sizes incur additional setup costs, the probable corrections (and hence reduced inaccuracy-induced inventory costs) at every order arrival make it more attractive to order less.

These results show that the determination of the optimal ordering quantity, even for this simple deterministic case, becomes considerably more complex in the presence of inaccuracies. We omit analysis of the case with under-registering of demand to avoid repetition, however we note that the insights that one can derive from that version of the model is similar to the ones discussed above. We refer the reader to Appendix A for details on the derivations.

\section{Simulation results}

\subsection{Description of the simulation model}

To evaluate the economic impact of inventory record inaccuracies, we compare the performance (in terms of expected total costs and number of lost sales per unit time) of a system with perfect inventory data to that of a system with execution errors. For that purpose, we use a detailed discrete-event simulation model of both systems and collect statistics on the relevant performance measures. The use of simulation, as opposed to an analytical method such as Markov chains, has allowed more realistic modeling of the inventory control system and the execution errors that happen at demand arrival epochs. Additionally, we were able to model and observe the effects of the correction opportunities defined above.

An important aspect of the simulation model is the modeling of execution errors. One of the contributions of this study is the use of a fairly general stochastic model of error occurrence upon arrival of a demand. As stated before, we considered errors that can occur in both directions of over-recording and underrecording of demand. As a result of these errors, the inventory records may show an inventory level that is higher or lower than the actual stock level. This constitutes a fundamental difference between our model and that considered in (Kang and Gershwin, 2005).

We assumed that demand arrivals are Poisson distributed. Since the recording error can be a function of the number of items demanded by a customer, we considered two cases based on the number of items demanded at each demand arrival. For the unit demand case, we assumed that upon a demand arrival, the sale of the unit goes unregistered with probability $p_{1}$, registered as two units with probability $p_{2}$, and is registered correctly (as one unit) with probability $\left(1-p_{1}-p_{2}\right)$. Note that the error level, which we define as $p_{1}+p_{2}$, corresponds to the average fraction of demand that is "misrecorded" in the long run. For the case of batch demand, we assumed that the number of items demanded by a customer is a random variable distributed according to a discrete uniform distribution (i.e., demand is compound Poisson distributed). In this case, it is possible to model execution errors in a number of different ways. Since the insights that can be gained from the model do not change drastically, we elected to use a simple form of error and followed a similar scheme to the one used in the unit demand case. That is, the entire sale goes unregistered with probability $p_{1}$, is recorded as twice the actual number of units sold with probability $p_{2}$, and is registered correctly with probability $\left(1-p_{1}-p_{2}\right)$.

The simulation model enabled a detailed modeling and implementation of correction opportunities. Since the simulation model keeps track of the actual inventory as well as the stock levels indicated by the IT system, instances of $\mathrm{CO}-1$ and $\mathrm{CO}-2$ could be detected. Upon occurrence of CO-1, the IT inventory records are corrected as zero. Upon occurrence of $\mathrm{CO}-2$, the sale is completed, and the inventory records are modified to allow for the sale. Recall that CO-2 only offers a partial correction of the inventory records, while CO-1 brings the recorded inventory levels to the actual.

The $Q$ and $r$ values to be used in the simulation experiments were determined using the approximation given in Hopp and Spearman (1996). The approximation uses the EOQ as $Q$ and computes the corresponding optimal $r$. Note that the use of the 
approximate $(Q, r)$ values is not critical to our results; hence, we elected to use a simple and effective inventory control policy, which is likely to be used in real-life applications. In the case of batch demand, $r$ values were determined by a simple search procedure, which evaluates the objective function value for different $r$ values and selects the best one.

Using the simulation model, we collect statistics on several performance measures. In particular, we calculate the average number of lost sales per year, the average total annual cost (i.e., sum of the annual holding, ordering, and lost sale costs) as well as the frequency of $\mathrm{CO}-1$ and $\mathrm{CO}-2$ occurrences.

The simulation model considered is a terminating simulation, where we simulate the inventory system for five years. We take 200 replications for each run. We do not show the confidence intervals generated (other than in Section 4.2) as most confidence intervals were sufficiently narrow. We do not employ the common random number technique as we usually compare different structures (with correction or without correction) and think that 200 replications will yield sufficient results. The results computed for the terminating simulation are averaged as per year, in the expectation that the resulting figure will be as unbiased as possible (with respect to starting and ending conditions). We should note that the non-stationary behavior induced by the terminating simulation turned out to be very mild; i.e., the differences between the first and last year's performance measure values were not unreasonably different.

\subsection{Insights from a typical case without correction opportunities}

A number of test runs on a typical case were carried out to understand the sensitivity of the results to various parameters. In these runs, we did not implement corrective actions upon CO-1 and CO-2 incidences, since we wanted to show some peculiarities of not modeling these events when assessing impact of execution errors. The set of parameters utilized in these runs are $c=\$ 50.00$, $D=21,900$ units per year, $K=\$ 15$ per order, $L=2$ days, annual holding rate, $i=30 \%$, and unit lost sale cost, $s=\$ 2.5$ per unit.

In all these runs, we assumed that the probability of overregistering and under-registering of demand are equal; that is, $p_{1}=p_{2}=p / 2$. Given the type of recording errors considered, this is the most interesting case, since in the long run, the total demand rate observed by the IT system is equal to the actual demand rate. This observation may lead the reader to conclude that average inventory levels should be stable over time due to the fact that the over-recording and under-recording errors are symmetric. However, note that in our model stockouts result in lost sales (as opposed to backlogging assumed by Kok and Shang (2007)). Consider a demand epoch at which the actual stock is zero and the recorded inventory level is positive. Our simulation model tallies this as a lost sale incident, but does not change the recorded inventory, since the sale does not get registered at the POS. In fact, the IT system sees no sales activity until the order arrives and the actual stock level increases above zero.

The untimely triggering of orders along with the lost sales assumption means that on the average, less than expected number of items are sold in each cycle. However, inventory is replenished without the IT system observing the lost sales. Hence, when no corrections (such as cycle counts and corrective actions upon CO-1 and CO-2 incidences) are performed, an inventory buildup is expected to occur over time. As there are no correction opportunities utilized in these runs, the average total annual cost will keep increasing indefinitely due to the slight increase in inventory level. Since the system never reaches steady state, we considered the average total annual cost during the first five years.

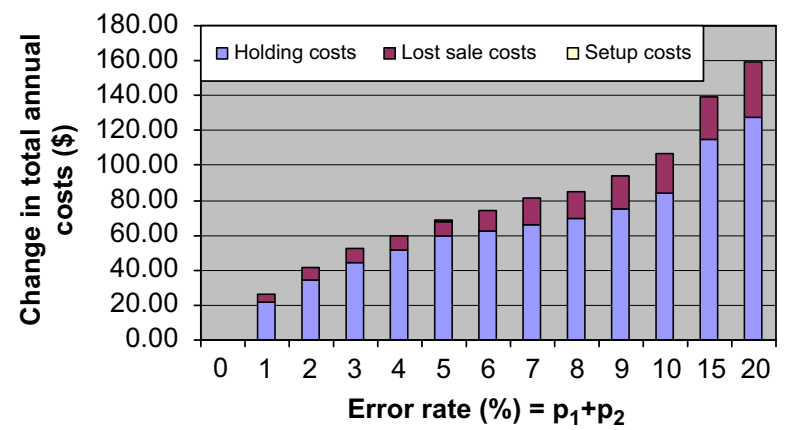

Fig. 4. Difference between the expected total annual costs of a system with errors and a system with perfect data, as a function of error level.

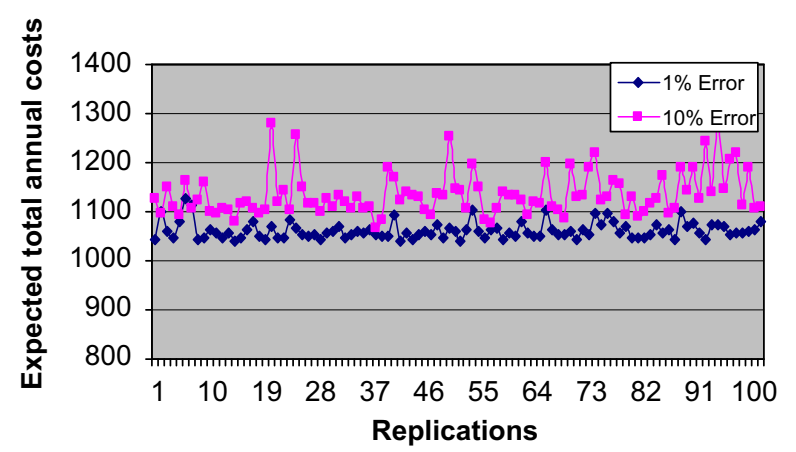

Fig. 5. Variability of total annual costs in typical case for $1 \%$ and $10 \%$ error level.

Effect of execution errors on different cost components: Fig. 4 shows the difference between the expected total annual costs of a system with execution errors and a system with perfect data, as a function of the error rate, $p$. As expected, the change in total costs increases as $p$ increases. We see that the difference in holding cost represents the majority of the cost difference; that is, among the three cost components considered, the holding cost is the one most severely affected due to the buildup of inventory. We don't observe any significant change in setup costs since the average number of orders per unit time in the long run is the same as long as $p_{1}=p_{2}=p$. Note that in our analysis, we did not consider costs such as obsolescence and spoilage. When one considers such costs, it is clear that the economic impact of the error becomes even more severe.

Effect of error on the variance of the total cost: Fig. 5 shows the expected total annual cost for error level, $p=1 \%$ and $p=10 \%$. We see that the expected total annual costs are a lot more volatile for the $10 \%$ error case. This shows that in addition to increases in the expected total annual cost, companies suffering from inventory inaccuracies also face increased variability in their inventory related costs, increasing their risks.

Dynamics of Error: Table 1 shows the 95\% confidence interval for the number of lost sales in each of the five simulated years, for different error levels. An interesting observation from Table 1 is that while the number of lost sales in each year is stationary for the system with perfect data (i.e., $p=0$ ), the number of lost sales decreases for systems with execution errors (i.e., $p>0$ ). At first glance, this may seem like a counterintuitive result, but the reason for this observation is that when inventory records are not corrected, the inaccuracies lead to an inventory buildup and hence, a decrease in the number of lost sales per year. However, because the holding costs of the system with execution errors increase disproportionately, the percent deviation of total cost from optimal cost increases each year. 
Table 1

95\% CI of the number of lost sales during each of the first five years for a typical case.

\begin{tabular}{|c|c|c|c|c|c|}
\hline \multirow[t]{2}{*}{ Percent Error Level, $p$} & \multicolumn{5}{|c|}{$95 \% \mathrm{CI}$ of number of lost sales during the indicated year } \\
\hline & Year 1 & Year 2 & Year 3 & Year 4 & Year 5 \\
\hline 0 & $2.90 \pm 0.93$ & $2.80 \pm 0.88$ & $2.93 \pm 0.85$ & $2.86 \pm 0.77$ & $2.93 \pm 0.93$ \\
\hline 1 & $6.69 \pm 1.58$ & $5.54 \pm 1.79$ & $4.07 \pm 1.51$ & $4.16 \pm 1.55$ & $3.89 \pm 1.21$ \\
\hline 5 & $11.58 \pm 2.65$ & $6.84 \pm 2.08$ & $4.42 \pm 1.69$ & $3.62 \pm 1.87$ & $3.26 \pm 1.35$ \\
\hline 10 & $21.67 \pm 4.09$ & $14.17 \pm 4.19$ & $8.64 \pm 3.22$ & $8.74 \pm 3.53$ & $7.21 \pm 3.35$ \\
\hline 20 & $29.35 \pm 5.51$ & $15.05 \pm 4.49$ & $13.08 \pm 4.87$ & $12.53 \pm 4.87$ & $8.42 \pm 4.21$ \\
\hline
\end{tabular}

Table 2

95\% CI of number of lost sales during each of the first five years for compound Poisson demand (compounding distribution is discrete uniform between 1 and 5 ).

\begin{tabular}{|c|c|c|c|c|c|}
\hline \multirow[t]{2}{*}{ Percent Error Level, $p$} & \multicolumn{5}{|c|}{$95 \% \mathrm{CI}$ of number of lost sales during the indicated year } \\
\hline & Year 1 & Year 2 & Year 3 & Year 4 & Year 5 \\
\hline 0 & $3.76 \pm 2.19$ & $3.83 \pm 1.53$ & $3.07 \pm 1.85$ & $3.60 \pm 1.60$ & $3.58 \pm 1.70$ \\
\hline 1 & $8.12 \pm 2.35$ & $8.68 \pm 2.96$ & $5.05 \pm 2.12$ & $5.91 \pm 2.64$ & $5.47 \pm 1.82$ \\
\hline 5 & $19.69 \pm 4.22$ & $10.17 \pm 3.80$ & $10.10 \pm 3.35$ & $7.19 \pm 3.73$ & $7.07 \pm 3.16$ \\
\hline 10 & $32.48 \pm 7.09$ & $22.49 \pm 6.57$ & $13.25 \pm 4.67$ & $13.06 \pm 4.64$ & $11.49 \pm 4.46$ \\
\hline 20 & $45.45 \pm 7.68$ & $31.03 \pm 8.76$ & $21.30 \pm 8.33$ & $17.48 \pm 6.71$ & $14.85 \pm 6.27$ \\
\hline
\end{tabular}

This indefinite inventory buildup is not observed in real-life systems since they get corrected periodically via various inspection procedures and cycle counting. To reflect this reality better, we incorporated correction opportunities and cycle counts in our simulation experiments presented in Section 4.3.

Effect of batch demand: As expected, the effects discussed above become more prominent when multiple units can be demanded by a customer. As an example, consider Table 2, which summarizes the number of lost sales when a compound Poisson distribution is used to model demand. Note that the mean demand generated by the compound Poisson distribution is the same as that generated by the unit Poisson distribution case so that the results from the two scenarios are comparable.

Note that the average lost sales numbers increase approximately by a factor of two under these conditions. The reason for this is that while in the case of compound Poisson demand the total number of stockout incidences is the same, the number of demands lost per demand arrival incidence is higher.

\subsection{Experimentation}

\subsubsection{Description of the test bed}

For the unit demand case, we identify a number of distinct cases by varying the relative values of the unit holding, setup, and lost sales costs as well as the length of lead time. In particular, we hold the holding cost, $h$ at $\$ 1.00$ per unit per year and vary $L, K / h$ ratio and $s / h$ ratio to design a set of experiments resulting in a total of 27 test cases. We present the parameters as well as the $Q$ and $r$ values used in each case in Table 3. To model a high-demand environment, we keep $D=21,900$ units per year. For each of the 27 cases, we consider four different values of error rate, $p: 1 \%, 5 \%$, $10 \%$ and $20 \%$.

For the batch demand case, we identify 18 distinct cases by varying the relative values of the unit holding, setup, and lost sales costs, as well as the parameters of the compounding distribution. We keep the lead time at 10 days for this set of experiments. In order to determine the effect of variability of the number of items demanded by each customer, we consider two different sets of experiments for the same mean demand. In the first set (Cases C1 through C9) the number of items demanded is sampled from 5,6 and 7 with equal probability of $1 / 3$. This configuration has a mean of 6 , and a variance of
Table 3

Test bed for the unit demand case.

\begin{tabular}{llllll}
\hline Case ID & $L$ & $K / h$ & $s / h$ & $Q$ & $r$ \\
\hline U1 & 1 & 10 & 1 & 662 & 76 \\
U2 & 1 & 10 & 10 & 662 & 82 \\
U3 & 1 & 10 & 20 & 662 & 84 \\
U4 & 1 & 30 & 1 & 1146 & 73 \\
U5 & 1 & 30 & 10 & 1146 & 81 \\
U6 & 1 & 30 & 20 & 1146 & 83 \\
U7 & 1 & 100 & 1 & 2093 & 71 \\
U8 & 1 & 100 & 10 & 2093 & 79 \\
U9 & 1 & 100 & 20 & 2093 & 81 \\
U10 & 10 & 10 & 1 & 662 & 647 \\
U11 & 10 & 10 & 10 & 662 & 668 \\
U12 & 10 & 10 & 20 & 662 & 674 \\
U13 & 10 & 30 & 1 & 1146 & 641 \\
U14 & 10 & 30 & 10 & 1146 & 664 \\
U15 & 10 & 30 & 20 & 1146 & 670 \\
U16 & 10 & 100 & 1 & 2093 & 633 \\
U17 & 10 & 100 & 10 & 2093 & 658 \\
U18 & 10 & 100 & 20 & 2093 & 664 \\
U19 & 30 & 10 & 1 & 662 & 1881 \\
U20 & 30 & 10 & 10 & 662 & 1917 \\
U21 & 30 & 10 & 20 & 662 & 1927 \\
U22 & 30 & 30 & 1 & 1146 & 1870 \\
U23 & 30 & 30 & 10 & 1146 & 1910 \\
U24 & 30 & 30 & 20 & 1146 & 1920 \\
U25 & 30 & 100 & 1 & 2093 & 1858 \\
U26 & 30 & 100 & 10 & 2093 & 1900 \\
U27 & 30 & 100 & 20 & 2093 & 1911 \\
\hline & & & & & \\
\hline
\end{tabular}

$(1 / 3)\left[(5-6)^{2}+(6-6)^{2}+(6-7)^{2}\right]=2 / 3$. In the second set of experiments, the number of items demanded is sampled from $2,4,6,8$, and 10 with equal probability of $1 / 5$. While this configuration has the same mean of 6 , it has a higher variance of 8 . To ensure that the results for these experiments are comparable to those found from the unit demand case, we reduce the arrival rate to 3650 per year so that the overall average demand stays the same. We present the parameters used in each case in Tables 4 and 5 .

We understand that one can perform more numerical tests, however the above described test problems are sufficient to yield results that can be interpreted. Note that all of the cases that we consider have high fill rates of above $99 \%$. In this respect, the 
Table 4

Test bed for the batch demand case with low variability ( mean=6, variance $=2 / 3$ ).

\begin{tabular}{llllll}
\hline Case ID & $L$ & $K / h$ & $s / h$ & $Q$ & $r$ \\
\hline C1 & 10 & 10 & 1 & 662 & 730 \\
C2 & 10 & 10 & 10 & 662 & 795 \\
C3 & 10 & 10 & 20 & 662 & 800 \\
C4 & 10 & 30 & 1 & 1146 & 705 \\
C5 & 10 & 30 & 10 & 1146 & 775 \\
C6 & 10 & 30 & 20 & 1146 & 785 \\
C7 & 10 & 100 & 1 & 2093 & 660 \\
C8 & 10 & 100 & 10 & 2093 & 770 \\
C9 & 10 & 100 & 20 & 2093 & 775 \\
\hline
\end{tabular}

Table 5

Test bed for the batch demand case with high variability ( $m e a n=6$, variance $=8$ ).

\begin{tabular}{llllll}
\hline Case ID & $L$ & $K / h$ & $s / h$ & $Q$ & $r$ \\
\hline C11 & 10 & 10 & 1 & 662 & 728 \\
C12 & 10 & 10 & 10 & 662 & 808 \\
C13 & 10 & 10 & 20 & 662 & 810 \\
C14 & 10 & 30 & 1 & 1146 & 715 \\
C15 & 10 & 30 & 10 & 1146 & 773 \\
C16 & 10 & 30 & 20 & 1146 & 785 \\
C17 & 10 & 100 & 1 & 2093 & 686 \\
C18 & 10 & 100 & 10 & 2093 & 740 \\
C19 & 10 & 100 & 20 & 2093 & 785 \\
\hline
\end{tabular}

results are biased so that we can see the effects under desired service levels in such systems.

\subsubsection{Effects of error on total cost}

Table 6 summarizes, for the unit demand case, the average differences in total annual costs of a system with execution errors and a system with perfect data in terms of "percent deviation," which is equal to the difference between the expected total annual costs of these systems divided by the expected total annual cost of the system with perfect data. Note that percent deviations are always positive, since the expected total annual cost of a system with execution errors is always higher than that of a system with perfect data. In addition to the information in Table 6 , detailed results for the unit demand and the batch demand cases are presented in Appendix B, in Tables 8, 9, and 10, 11 , respectively. The values in Table 6 are determined by computing the average across all relevant test cases. We should note that the standard deviation of the average costs observed in the simulation runs (which are used to generate the percent deviations in Table 6 ) are generally less than $5 \%$ of the cost value.

We see from Table 6 that the average percent deviations are quite significant in general (about 10-12\%), and can even reach as high as $50 \%$. When one translates the percent values to absolute dollar figures, it is clear that a significant cost is incurred by retailers due to these errors. Our analytical results are also backed by empirical research; Raman et al. (2001) attribute an equivalent of $10 \%$ of the profits of the organization they studied as a rough estimate of the losses associated with inaccuracies. We make the following further observations from Table 6.

- We see that for small lead times, the average percent deviation is higher. This is due to the fact that small lead times imply relatively lower reorder levels since reorder levels are set as the sum of the average demand during lead time and a safety
Table 6

Average and maximum percent deviation of the actual cost from the no-error case for the unit Poisson demand case.

\begin{tabular}{llll}
\hline Tested factors & $\begin{array}{l}\text { Factor } \\
\text { levels }\end{array}$ & $\begin{array}{l}\text { Average percent } \\
\text { deviation from } \\
\text { the no-error case }\end{array}$ & $\begin{array}{l}\text { Maximum percent } \\
\text { deviation from the } \\
\text { no-error case }\end{array}$ \\
\hline Error level, $p\left(p=p_{1}=p_{2}\right)$ & 0.01 & 2.56 & 7.79 \\
& 0.05 & 6.16 & 16.74 \\
& 0.10 & 10.91 & 32.14 \\
& 0.20 & 15.94 & 47.43 \\
Lead time, $L$ & 1 & 11.60 & 47.43 \\
& 10 & 7.86 & 31.78 \\
Lost sales to holding & 30 & 7.22 & 25.36 \\
cost ratio, $s / h$ & 1 & 4.78 & 15.50 \\
& 10 & 8.77 & 32.44 \\
Setup cost to holding & 20 & 13.13 & 47.43 \\
cost ratio, $K / h$ & 10 & 12.82 & 47.43 \\
& 30 & 9.23 & 30.09 \\
& 100 & 4.63 & 18.76 \\
\hline
\end{tabular}

stock to protect against variability of demand during lead time. As an example, observe that the reorder levels for the cases with a lead time of one day are about 80 units, whereas the reorder levels for the cases with a lead time of ten days are about 660 units. Higher reorder levels (or equivalently, higher safety stock levels) serves as a buffer against stockouts associated with the erroneous (possibly delayed) triggering of replenishment orders due to inventory data inaccuracies. Clearly, the increase in lead times and reorder levels leads to higher inventory holding costs, and in cases where holding costs are relatively high compared to lost sales costs, this overall trend may not be seen. For example, consider cases with $s / h=1$ (case ID numbers U1, U4, U7, U10, U13, U16, U19, $\mathrm{U} 22$, and $\mathrm{U} 25$ ). We see that the average percent deviation for cases with $L=1, L=10$, and $L=30$ days are $\% 5.57, \% 3.50$, and $\% 5.28$, respectively.

- As the unit lost sale cost, $s$, increases the average percent deviation from the no-error case increases. This is trivial since stockouts that occur due to untimely triggering of replenishment orders become more and more expensive as the lost sale cost increases. Note that even sizable increases in the $s / h$ ratio does not lead to dramatic increases in the reorder levels. Hence, the average number of lost sales are comparable between these cases with $s / h=1, s / h=10$, and $s / h=20$. However, the impact on the overall cost values differs significantly due to the much higher unit lost sale cost.

- Low setup cost to holding cost ratios and high lost sales cost to holding cost ratios again lead to increased percent deviations. Low setup cost to holding cost ratios lead to small $Q$, meaning that all other things being equal, more frequent orders need to be triggered. This leads to more opportunities for making untimely orders, which explains the increased average percent deviations. Similar arguments can be made to explain the behavior of other factors.

When customers may request more than one item at a time, the impact of errors becomes much more significant. While the overall behavior of the percent deviations were very similar to those in the unit demand cases, the magnitude of the average percent deviation in total costs increased to $18.90 \%$ from $9.74 \%$. Furthermore, the average percent deviation increased to $22.57 \%$ for the cases with higher demand variability (case ID numbers C11 through C19). 


\subsubsection{Number of correction opportunities}

It is important to know the number of correction opportunities since each incidence may provide an opportunity for correction of inventory records. Table 7 summarizes the number of $\mathrm{CO}-1$ and $\mathrm{CO}-2$ incidences in the experiments.

The first observation from Table 7 is that the average number of correction opportunities increases with the increasing error rate for the unit demand case. In the batch demand case, $\mathrm{CO}-1$ and $\mathrm{CO}-2$ values should be compared with the third row of the table, error level of $10 \%$. (Actually, they should be compared with the averages of Cases U10-U18, as they are the cases with the same parameters. However, the remarks would be similar.) Note that the number of correction opportunities is less as the number of customer arrivals in the batch demand (compound Poisson) case is one-third of the unit demand case. In that respect, the fraction of customers creating a chance for a correction opportunity (either CO-1 or CO-2) is at least doubled in the case of batch demand.

The maximum number of CO- 1 and CO-2 incidences (and the ID of the case that attains them) are provided in column 3 and column 5 of Table 7, respectively. In almost all error levels, Case $\mathrm{U} 1$ attains the maximum number of $\mathrm{CO}-1$ incidences. It is interesting to see that Case $\mathrm{U} 1$ corresponds to $L=1, K=10, s=1$, (i.e., the shortest lead time, the smallest fixed ordering cost, and the smallest unit lost sale cost). This is expected because Case U1 has a low reorder level and allows for frequent ordering. In batch demand case, the maximum number of $\mathrm{CO}-1$ incidences occur for the case where we have $K=10$, and $s=1$ (or $K=100, s=1$ ), which imply a high number of lost sales.

When we look for the maximum statistics for $\mathrm{CO}-2$, the results are a bit different. In almost all error levels, Case U22 or Case U25 attains the maximum number of $\mathrm{CO}-2$ incidences. Case U22 corresponds to $L=30, K=30, s=1$, and Case U27 corresponds to $L=30, K=100, s=1$ (i.e., the longest lead-time, a large fixed ordering cost, and the smallest unit lost sale cost). In these cases, the system has a tendency of ordering less frequently, which results in more lost sales on the books than the actual numbers (definition of $\mathrm{CO}-2$ ). In batch demand case, the maximum number of CO-2 incidences occur for the case where we have $K=100$, and $s=1$, similarly to the unit demand case.

Fig. 6 shows the average number of $\mathrm{CO}-1$ and $\mathrm{CO}-2$ incidences per year (averaged over five years) as a function of the reorder level, for Case U22. In order to obtain these results, we use a simulation experiment for each reorder level used, and run a transient simulation for five years, with 200 replications. The optimal reorder level, $r^{*}$ is also shown. We see that the average number of CO-1 incidences per year is quite low, regardless of the reorder level used. On the other hand, the average number of CO-2 incidences per year can be significant. Note that both of them are decreasing as the reorder level increases. This makes sense, since as the reorder level increases, the likelihood that the actual stock or the inventory records hits zero decreases.
An important observation from the experiments is that the number of correction opportunities and especially $\mathrm{CO}-2$, is very sensitive to the reorder level. Small increases in the reorder level cause the CO-2 occurrences to drop sharply. Note that this result implies that if one is operating a lean system (i.e., low cycle stock and safety stock levels) with possible recording errors, the number of opportunities to correct the records is increasing, and hence the importance of using this information becomes more apparent.

\subsubsection{Effect of cycle counts}

To gain insight on the effectiveness of cycle counts in decreasing the inaccuracy, we observed how total annual cost improves as a function of different audit frequencies. For this case, we use a transient simulation for five years, with 200 replications, and we do not implement $\mathrm{CO}-1$ or $\mathrm{CO}-2$ for these simulations. Fig. 7 shows the expected total annual costs (averaged over five years) as a function of annual audit frequency for the typical case discussed in Section 4.2. Note that the total annual costs shown in

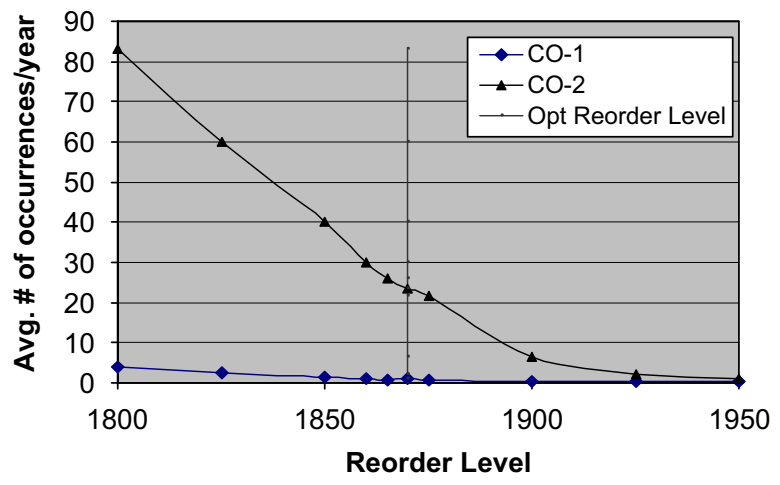

Fig. 6. Average annual CO- 1 and CO-2 incidences for Case U22.

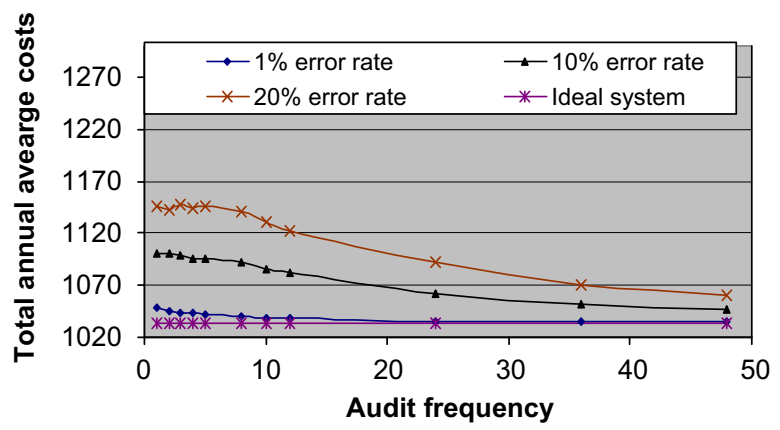

Fig. 7. Actual total annual costs (averaged over five years) as a function of audit frequency.

Table 7

Summary results for $\mathrm{CO}-1$ and $\mathrm{CO}-2$ in various experiments.

\begin{tabular}{|c|c|c|c|c|}
\hline \multirow[t]{2}{*}{ All cases } & \multicolumn{2}{|c|}{ Mean number of $\mathrm{CO}-1$ incidences per year } & \multicolumn{2}{|c|}{ Mean number of $\mathrm{CO}-2$ incidences per year } \\
\hline & Average & Max (Case ID) & Average & Max (Case ID) \\
\hline Unit Poisson with $1 \%$ error ( 27 cases) & 0.37 & $0.85(\mathrm{U} 4)$ & 1.50 & $5.46(\mathrm{U} 25)$ \\
\hline Unit Poisson with $5 \%$ error ( 27 cases) & 0.43 & $0.86(\mathrm{U} 1)$ & 2.86 & 11.31(U25) \\
\hline Unit Poisson with $10 \%$ error ( 27 cases) & 0.69 & 1.40 (U1) & 3.55 & $14.60(\mathrm{U} 22)$ \\
\hline Unit Poisson with $20 \%$ error ( 27 cases) & 0.73 & 1.50 (U1) & 5.62 & 20.46 (U22) \\
\hline Compound Poisson (low variability) $10 \%$ error ( 9 cases) & 0.59 & $1.31(\mathrm{C} 7)$ & 2.97 & $12.08(\mathrm{C} 7)$ \\
\hline Compound Poisson (high variability) $10 \%$ error ( 9 cases) & 0.63 & $1.06(\mathrm{C} 11)$ & 2.97 & 8.22 (C17) \\
\hline
\end{tabular}


the figure for the "ideal system" correspond to the expected total annual costs that can be achieved by a system with perfect data.

From Fig. 7, we see that it is possible to close the gap between the total annual cost incurred by a system with execution errors and that incurred by a system with perfect data, by conducting a sufficiently high number of inventory audits. In our runs, this sufficient audit frequency corresponded to about 50, or about every week. Furthermore, we can observe that for high error rates (e.g., 20\%, which is a modest estimate for errors in real-life systems) low audit frequencies do not offer a significant improvement in system costs. When one also considers the additional cost of conducting these inventory audits, our results echo the observation that inventory audits alone are not sufficient to control the inaccuracies and their effects in a cost-effective manner.

\section{Managerial insights}

The following summarize the main managerial insights derived from our study:

- The economic impact of inventory record inaccuracies can be significant. The percent deviation is particularly high for systems with small order sizes and low reorder levels. Low reorder levels imply that when data inaccuracies exist, stockout costs in the actual system increase, since orders are not triggered at the right time. Similarly, with small order sizes more frequent orders need to be triggered, which leads to more opportunities, on average, of making untimely orders.

- In addition to incurring higher costs, systems suffering from record inaccuracies are subject to more risk, since variability of inventory-related costs also increase as the error level increases.

- The economic impact of execution errors are likely to be much more severe in systems with batch demand, particularly when the number of items demanded by each customer is highly variable.

- When the errors are left uncorrected, cost performance deteriorates with time, due to the inventory buildup. This observation explains the fact that most real-life systems (including those with very sophisticated tracking sensors) implement various forms of inventory audit mechanisms to keep inaccuracies under control.

- In general, low inventory audit frequencies are only partially effective in controlling the economic impact of record inaccuracies. The effectiveness of inventory audits increases as more and more audits are performed. However, one should recognize that inventory audits, such as cycle counting, are expensive and disruptive.

- Since cycle counting is only partially effective in eliminating the impact of inaccuracies, companies should make effective use of the information obtainable through naturally occurring correction opportunities. We have identified two such events in this study.

In addition to making effective use of correction opportunities and other types of free or cheap forms of information, companies that observe significant levels of inventory record inaccuracies should consider various actions to prevent these errors. These actions can be training of workers at the cash registers so that the correct bar codes are scanned, better labeling of products and boxes, improved shelving schemes that minimize picking errors, reduction in the number of SKUs to minimize errors, etc.

In parallel to prevention and correction activities, companies should seek ways to implement better inventory control policies.
Our simple model in Section 3.2 demonstrated that even under simplistic assumptions, the expressions for optimal ordering quantities can become quite complex, due to the different types of trade-offs that one needs to consider. In our model, for example, in addition to the trade-offs between holding, setup and lost sales costs, one should consider the impact of these decisions on data quality. If, for example, errors are likely to be corrected at order delivery epochs, one may want to reduce the order size (or reorder level) to take advantage of increased record correction capability.

\section{Conclusions}

Motivated by recent empirical evidence, we considered the economic impact of inventory record inaccuracies that arise due to execution errors. In contrast to prior work on the subject, we paid particular attention to the modeling of execution errors and modeled a set of probable events regarding the erroneous registering of sales at each demand arrival. To evaluate the economic impact of inventory record inaccuracies, we used a simulation model, which allowed us to model various types of complex behavior and execution errors. We considered a continuous-review inventory system with lost sales, controlled by the well-known $(Q, r)$ policy, and compared the performance of the system with inaccuracies to that of an ideal system with perfect data.

In addition to observing the impact (and potential) of correction opportunities through simulation experiments, we used a simple inventory control model to show the effect of probabilistic correction on the expected total cost and the optimal ordering quantity. We found that the incorporation of correction opportunities to automated correction schemes holds a lot of premise for management of inventory systems with data inaccuracies, since these can improve the accuracy of the records at no cost (or at much lower cost compared to traditional inventory auditing methods).

One of the conclusions we can draw from this study is that inventory control decisions that consider the existence of recording inaccuracies can be complicated. For instance, if one observes Eq. (A.7), the optimal ordering quantity is a function of the reorder level, as well as other pertinent problem parameters. This is surprising since, in a traditional, deterministic setting, there is no effect of the reorder level in setting the EOQ. However, the setting with recording errors with a probabilistic correction mechanism leads to a more complicated relation.

We note that inventory systems with recording errors and correction opportunities need to be studied carefully before serious investment decisions are made to improve the information infrastructure. Such work can be used to determine the "real value" of technologies like RFID tags. Many experts argue that even with RFID tags there will be data inaccuracies. Hence, it is important to determine mechanisms through which the system can implement automated corrections.

To summarize, managing inventory data quality in today's complex supply chains is a critical function for companies. In addition to affecting the inventory-related costs, inventory record inaccuracies are likely to impact the performance of the whole supply chain. Inaccuracies are likely to introduce errors into the forecasting process, since supply chain forecasts are generally based on POS data, which can be erroneous due to execution errors. Additionally, inaccuracies and their eventual correction are likely to increase the bullwhip effect by increasing the variability of orders. The impact of execution errors and other types of inventory record inaccuracies on the overall supply chain 
performance needs to be studied further to understand the various trade-offs.

\section{Appendix A}

We first derive the total actual cost for $n$ cycles for the inventory system with no corrections. Let $I_{A}\left(t_{n}\right)$ denote the level of actual inventory on-hand at the end of the $n$th cycle. The cycles, which are marked by order arrivals, are indicated in Fig. 1. Then, $I_{A}\left(t_{n}\right)$ can be calculated as

$I_{A}\left(t_{n}\right)=Q+n(Q-r) \frac{p}{1+p}$

The average inventory per unit time for the $n$th cycle is equal to

$\bar{I}_{A}(n)=\frac{Q}{2}+\frac{2 n+1}{2}(Q-r) \frac{p}{1+p}$.

Hence, the total actual cost for $n$ cycles, $T C(n, Q, p)$ is equal to the sum of order setup, variable purchasing and holding costs, as follows.

$$
\begin{aligned}
T C(n, Q, p) & =\sum_{j=1}^{n}\left(K+c Q+h \frac{Q+r p}{D(1+p)}\left[\frac{Q}{2}+\frac{2 n+1}{2}(Q-r) \frac{p}{1+p}\right]\right) \\
& =n f_{1}(Q, p)+n^{2} f_{2}(Q, p),
\end{aligned}
$$

where

$$
\begin{aligned}
& f_{1}(Q, p)=K+c Q+\frac{h Q}{2}\left(\frac{Q+r p}{D(1+p)}\right) \text { and } \\
& f_{2}(Q, p)=h \frac{Q+r p}{D(1+p)}\left[\frac{(Q-r) p}{2(1+p)}\right] .
\end{aligned}
$$

Note that Eq. (A.3) does not include any cost terms for lost sales. This is due to the fact that in the case of deterministic demand, the actual system does not register any lost sales even when the IT system shows an inventory level of zero, as in Fig. 1.
To derive the expected total cost for the system with periodic correction of inventory records, we first derive the expression for the total cost for $n$ cycles, given that a correction occurs in the $n$th cycle. Recall that $K_{q}$ denotes the cost of the corrective action. Adding this cost to the total cost for $n$ periods, we get the total cost function for the case with corrections, denoted by $T C_{W C}(n, Q, p, q)$, as

$T C_{W C}(n, Q, p, q)=n f_{1}(Q, p)+n^{2} f_{2}(Q, p)+n K_{q}$

This probabilistic model of correction of the inventory records allows us to define renewal intervals during which the process statistically repeats itself. Hence, we can calculate the expected average cost per unit time, $E[G(Q p, q)]$ as follows

$E[G(Q, p, q)]=\frac{D(1+p)}{Q+r p} \sum_{n=1}^{\infty} P(N=n)\left(\frac{T C_{W C}(n, Q, p, q)}{N} \mid N=n\right)$

where $N$ represents the random number of inventory cycles in a renewal cycle. It is trivial to observe that $N$ is geometrically distributed, i.e., $P(N=n)=q(1-q)^{n-1}$. Then, Eq. (A.5) can be rewritten as

$$
\begin{aligned}
E[G(Q, p, q)]= & \frac{D(1+p)}{Q+r p} \sum_{n=1}^{\infty} \frac{n f_{1}(Q, p)+n^{2} f_{2}(Q, p)+n K_{q}}{n} q(1-q)^{n-1} \\
= & \frac{D(1+p)}{Q+r p}\left[\left(K_{q}+f_{1}(Q, p)\right) \sum_{n=1}^{\infty} q(1-q)^{n-1}\right. \\
& \left.+f_{2}(Q, p) \sum_{n=1}^{\infty} n q(1-q)^{n-1}\right] \\
= & \frac{D(1+p)}{Q+r p}\left[K_{q}+f_{1}(Q, p)+\frac{f_{2}(Q, p)}{q}\right]
\end{aligned}
$$

\begin{tabular}{|c|c|c|c|c|c|c|c|c|c|c|c|c|}
\hline \multirow[t]{2}{*}{ Case } & \multicolumn{6}{|l|}{ 1\% Error } & \multicolumn{6}{|l|}{ 5\% Error } \\
\hline & $\begin{array}{l}\text { Exp. annual } \\
\text { cost }\end{array}$ & $\begin{array}{l}\text { Std. dev. of } \\
\text { annual cost }\end{array}$ & $\begin{array}{l}\% \\
\text { Dev. }\end{array}$ & $\begin{array}{l}\text { Actual fill } \\
\text { rate }\end{array}$ & $\begin{array}{l}\text { Avg. no. of } \\
\text { CO-1 }\end{array}$ & $\begin{array}{l}\text { Avg. no. of } \\
\text { CO-2 }\end{array}$ & $\begin{array}{l}\text { Exp. Annual } \\
\text { Cost }\end{array}$ & $\begin{array}{l}\text { Std. dev. of } \\
\text { annual cost }\end{array}$ & $\begin{array}{l}\% \\
\text { Dev. }\end{array}$ & $\begin{array}{l}\text { Actual fill } \\
\text { rate }\end{array}$ & $\begin{array}{l}\text { Avg. no. of } \\
\text { CO-1 }\end{array}$ & $\begin{array}{l}\text { Avg. no. of } \\
\text { CO-2 }\end{array}$ \\
\hline 1 & 698.92 & 13.20 & 2.69 & 99.98 & 0.74 & 1.80 & 727.15 & 24.79 & 6.84 & 99.97 & 0.86 & 2.62 \\
\hline 2 & 724.48 & 20.79 & 5.42 & 99.99 & 0.45 & 0.23 & 768.48 & 34.83 & 11.83 & 99.98 & 0.57 & 0.37 \\
\hline 3 & 742.24 & 39.33 & 7.79 & 99.99 & 0.38 & 0.10 & 803.81 & 69.61 & 16.74 & 99.98 & 0.51 & 0.16 \\
\hline 4 & 1178.27 & 12.60 & 1.48 & 99.97 & 0.85 & 2.39 & 1206.75 & 25.11 & 3.93 & 99.97 & 0.84 & 3.32 \\
\hline 5 & 1202.68 & 20.76 & 3.03 & 99.99 & 0.40 & 0.11 & 1253.77 & 41.28 & 7.41 & 99.98 & 0.50 & 0.31 \\
\hline 6 & 1220.31 & 40.82 & 4.36 & 99.99 & 0.34 & 0.07 & 1295.27 & 79.52 & 10.77 & 99.98 & 0.47 & 0.19 \\
\hline 7 & 2120.91 & 14.44 & 0.82 & 99.97 & 0.73 & 2.07 & 2148.75 & 24.30 & 2.14 & 99.96 & 0.75 & 3.18 \\
\hline 8 & 2148.80 & 25.34 & 1.86 & 99.99 & 0.40 & 0.27 & 2196.72 & 45.56 & 4.13 & 99.98 & 0.48 & 0.35 \\
\hline 9 & 2174.53 & 52.84 & 2.90 & 99.99 & 0.35 & 0.13 & 2252.52 & 102.38 & 6.59 & 99.98 & 0.45 & 0.23 \\
\hline 10 & 730.54 & 9.87 & 1.37 & 99.95 & 0.64 & 4.59 & 750.30 & 19.09 & 4.11 & 99.95 & 0.71 & 8.63 \\
\hline 11 & 769.04 & 27.16 & 3.94 & 99.99 & 0.23 & 0.42 & 800.76 & 36.19 & 8.23 & 99.98 & 0.28 & 0.87 \\
\hline 12 & 786.82 & 45.50 & 5.72 & 99.99 & 0.18 & 0.15 & 826.77 & 66.11 & 11.09 & 99.99 & 0.25 & 0.42 \\
\hline 13 & 1209.38 & 11.41 & 0.87 & 99.95 & 0.60 & 4.28 & 1228.01 & 19.55 & 2.43 & 99.95 & 0.63 & 8.32 \\
\hline 14 & 1245.97 & 29.48 & 2.15 & 99.99 & 0.20 & 0.45 & 1280.60 & 42.84 & 4.98 & 99.98 & 0.27 & 0.70 \\
\hline 15 & 1260.36 & 43.79 & 2.92 & 99.99 & 0.15 & 0.13 & 1311.32 & 77.73 & 7.08 & 99.99 & 0.22 & 0.28 \\
\hline 16 & 2143.71 & 8.73 & 0.39 & 99.95 & 0.57 & 4.87 & 2163.16 & 21.03 & 1.30 & 99.94 & 0.59 & 8.23 \\
\hline 17 & 2197.90 & 31.02 & 1.28 & 99.99 & 0.21 & 0.57 & 2233.59 & 45.13 & 2.93 & 99.98 & 0.25 & 0.82 \\
\hline 18 & 2211.20 & 62.85 & 2.22 & 99.99 & 0.15 & 0.33 & 2242.32 & 73.06 & 3.66 & 99.99 & 0.21 & 0.39 \\
\hline 19 & 775.54 & 12.43 & 0.99 & 99.94 & 0.49 & 4.26 & 791.02 & 20.23 & 3.01 & 99.93 & 0.53 & 9.44 \\
\hline 20 & 826.04 & 37.29 & 2.82 & 99.99 & 0.15 & 0.64 & 855.14 & 50.14 & 6.44 & 99.98 & 0.19 & 1.45 \\
\hline 21 & 845.76 & 58.29 & 4.03 & 99.99 & 0.11 & 0.41 & 886.11 & 83.30 & 8.99 & 99.99 & 0.15 & 0.77 \\
\hline 22 & 1260.91 & 11.03 & 0.51 & 99.92 & 0.53 & 4.63 & 1418.03 & 23.92 & 13.04 & 99.20 & 0.65 & 11.05 \\
\hline 23 & 1299.16 & 33.95 & 1.53 & 99.99 & 0.16 & 0.79 & 1331.72 & 49.29 & 4.07 & 99.98 & 0.17 & 1.39 \\
\hline 24 & 1320.40 & 56.96 & 5.23 & 99.99 & 0.11 & 0.41 & 1358.44 & 86.41 & 8.26 & 99.99 & 0.14 & 0.75 \\
\hline 25 & 2189.90 & 13.61 & 0.25 & 99.92 & 0.59 & 5.46 & 2203.69 & 17.57 & 0.89 & 99.91 & 0.59 & 11.31 \\
\hline 26 & 2243.83 & 43.87 & 1.05 & 99.99 & 0.14 & 0.61 & 2271.30 & 50.83 & 2.29 & 99.98 & 0.17 & 1.11 \\
\hline 27 & 2264.38 & 69.76 & 1.56 & 99.99 & 0.11 & 0.22 & 2298.00 & 84.30 & 3.07 & 99.99 & 0.13 & 0.43 \\
\hline
\end{tabular}

Note that for $q=0$ (i.e., correction probability of zero) the expected cost per unit time goes to infinity as expected, since the actual inventory on-hand keeps on accumulating when no correction is applied. In real life systems, the average cost per

Table 8

Detailed results for unit poisson demand with $1 \%$ and 5\% error levels. 
unit time is never infinity, since these systems are always corrected periodically through various means of correction, such as cycle counts, aisle walks, etc. Suppose the average time in between corrections for a system is equal to $\eta$. This can be modeled with an implied correction probability, which is equal to $q_{\eta}=1 / \eta$ to calculate the expected cost per unit time with the above equation.

Eq. (A.6) is the expected cost function when the system operates under a $(Q, r)$ policy with an existing error level, $p$ and periodic correction probability, $q$. A number of analytical properties of Eq. (A.6) can be deduced.

Property 1. Expected total cost is a decreasing function of reorder level, $r$.

Property 2. For constant $K_{q}$, the expected total cost function is a decreasing function of $q$. In other words, to operate, if feasible, with a greater periodic correction probability decreases the cost. However, $K_{q}$ is expected to be an increasing function of $q$.
Property 3. The expected total cost function is convex in $Q$. Hence, one can find the optimal value of $Q$ given that all the parameters can be estimated.

Using the above results, we can calculate the optimal $Q$ value for given values of error level, $p$, and correction probability, $q$. Note that in this calculation we assume that the reorder level, $r$, fixed. In reality, the reorder level will be a function of the ordering quantity, $Q$, when lead time is longer than the cycle length implied by the ordering quantity. Taking the derivative with respect to $Q$, we calculate the optimal order quantity to be equal to

$Q^{*}(p, q)=\sqrt{\frac{2\left(K+K_{q}-c r p\right) q D(1+p)^{2}}{h[q(1+p)+p]}}-r p$

Note that this function is not necessarily defined for all parameter values. However, one can show that there exists a range of values of $r$ where $Q^{*}$ will be positive.

Table 9

Detailed results for unit poisson demand with $10 \%$ and $20 \%$ error levels.

\begin{tabular}{|c|c|c|c|c|c|c|c|c|c|c|c|c|}
\hline \multirow[t]{2}{*}{ Case } & \multicolumn{6}{|l|}{$10 \%$ Error } & \multicolumn{6}{|l|}{$20 \%$ Error } \\
\hline & $\begin{array}{l}\text { Exp. annual } \\
\text { cost }\end{array}$ & $\begin{array}{l}\text { Std. dev. of } \\
\text { annual cost }\end{array}$ & $\begin{array}{l}\% \\
\text { Dev. }\end{array}$ & $\begin{array}{l}\text { Actual fill } \\
\text { rate }\end{array}$ & $\begin{array}{l}\text { Avg. No. of } \\
\text { CO-1 }\end{array}$ & $\begin{array}{l}\text { Avg. no. of } \\
\mathrm{CO}-2\end{array}$ & $\begin{array}{l}\text { Exp. annual } \\
\text { cost }\end{array}$ & $\begin{array}{l}\text { Std. dev. of } \\
\text { annual cost }\end{array}$ & $\begin{array}{l}\% \\
\text { Dev. }\end{array}$ & $\begin{array}{l}\text { Actual fill } \\
\text { rate }\end{array}$ & $\begin{array}{l}\text { Avg. no. of } \\
\text { CO-1 }\end{array}$ & $\begin{array}{l}\text { Avg. no. of } \\
\text { CO-2 }\end{array}$ \\
\hline 1 & 750.10 & 45.32 & 10.22 & 99.94 & 1.40 & 3.30 & 786.05 & 56.08 & 15.50 & 99.92 & 1.50 & 5.01 \\
\hline 2 & 833.78 & 59.17 & 21.33 & 99.96 & 1.01 & 0.49 & 910.15 & 85.85 & 32.44 & 99.94 & 1.09 & 0.96 \\
\hline 3 & 909.91 & 118.99 & 32.14 & 99.85 & 0.94 & 0.24 & 1015.20 & 177.73 & 47.43 & 99.95 & 1.02 & 0.48 \\
\hline 4 & 1229.36 & 44.80 & 5.88 & 99.93 & 1.35 & 3.93 & 1267.81 & 54.17 & 9.19 & 99.90 & 1.40 & 5.21 \\
\hline 5 & 1319.38 & 65.10 & 13.03 & 99.96 & 0.90 & 0.54 & 1405.77 & 99.33 & 20.43 & 99.93 & 0.99 & 0.81 \\
\hline 6 & 1413.88 & 129.68 & 20.91 & 99.96 & 0.82 & 0.22 & 1521.23 & 198.78 & 30.09 & 99.94 & 0.92 & 0.40 \\
\hline 7 & 2170.84 & 42.88 & 3.19 & 99.93 & 1.12 & 3.32 & 2207.64 & 55.33 & 4.94 & 99.90 & 1.13 & 4.26 \\
\hline 8 & 2276.12 & 75.52 & 7.89 & 99.95 & 0.83 & 0.29 & 2357.27 & 112.62 & 11.74 & 99.93 & 0.86 & 0.64 \\
\hline 9 & 2360.22 & 148.33 & 11.68 & 99.96 & 0.77 & 0.21 & 2509.81 & 257.73 & 18.76 & 99.93 & 0.78 & 0.39 \\
\hline 10 & 766.84 & 35.69 & 6.40 & 99.92 & 0.97 & 9.84 & 789.61 & 43.06 & 9.56 & 99.91 & 0.98 & 15.08 \\
\hline 11 & 850.93 & 62.01 & 15.01 & 99.97 & 0.50 & 1.05 & 907.14 & 81.35 & 22.60 & 99.96 & 0.55 & 2.77 \\
\hline 12 & 909.96 & 123.53 & 22.27 & 99.97 & 0.42 & 0.53 & 980.71 & 142.07 & 31.78 & 99.96 & 0.49 & 1.75 \\
\hline 13 & 1244.68 & 35.06 & 3.82 & 99.92 & 0.93 & 9.27 & 1270.83 & 41.54 & 6.00 & 99.90 & 0.91 & 14.38 \\
\hline 14 & 1335.53 & 70.43 & 9.49 & 99.96 & 0.45 & 1.02 & 1390.37 & 78.98 & 13.98 & 99.95 & 0.52 & 2.76 \\
\hline 15 & 1405.31 & 133.73 & 14.75 & 99.97 & 0.40 & 0.54 & 1479.55 & 163.87 & 20.81 & 99.96 & 0.44 & 1.44 \\
\hline 16 & 2183.20 & 37.93 & 2.24 & 99.91 & 0.85 & 9.29 & 2209.24 & 48.94 & 3.46 & 99.90 & 0.88 & 12.30 \\
\hline 17 & 2292.99 & 73.01 & 5.66 & 99.96 & 0.44 & 1.41 & 2360.45 & 102.86 & 8.77 & 99.94 & 0.48 & 1.88 \\
\hline 18 & 2335.96 & 122.60 & 7.99 & 99.97 & 0.39 & 0.60 & 2417.10 & 174.72 & 11.74 & 99.96 & 0.42 & 1.40 \\
\hline 19 & 803.82 & 32.93 & 4.67 & 99.90 & 0.79 & 11.78 & 821.32 & 42.12 & 6.95 & 99.89 & 0.86 & 19.51 \\
\hline 20 & 901.86 & 73.16 & 12.26 & 99.96 & 0.36 & 2.24 & 941.74 & 82.41 & 17.22 & 99.96 & 0.39 & 4.36 \\
\hline 21 & 944.31 & 126.84 & 16.15 & 99.97 & 0.27 & 1.30 & 1019.19 & 162.96 & 25.36 & 99.96 & 0.33 & 3.35 \\
\hline 22 & 1430.84 & 28.62 & 14.06 & 99.16 & 0.85 & 14.60 & 1447.16 & 33.25 & 15.36 & 99.14 & 0.87 & 20.46 \\
\hline 23 & 1371.40 & 72.20 & 7.17 & 99.97 & 0.31 & 1.99 & 1419.33 & 86.05 & 10.92 & 99.96 & 0.37 & 4.58 \\
\hline 24 & 1436.43 & 132.99 & 14.48 & 99.97 & 0.27 & 0.82 & 1480.08 & 137.86 & 17.96 & 99.97 & 0.31 & 3.05 \\
\hline 25 & 2213.29 & 29.28 & 1.33 & 99.89 & 0.74 & 14.34 & 2233.30 & 36.85 & 2.24 & 99.87 & 0.73 & 18.52 \\
\hline 26 & 2313.29 & 71.75 & 4.18 & 99.97 & 0.28 & 1.74 & 2361.54 & 86.21 & 6.35 & 99.96 & 0.33 & 3.99 \\
\hline 27 & 2373.02 & 124.68 & 6.44 & 99.97 & 0.23 & 0.88 & 2425.36 & 130.97 & 8.78 & 99.97 & 0.28 & 2.08 \\
\hline
\end{tabular}

Table 10

Detailed results for selected compound Poisson cases with 10\% error level.

\begin{tabular}{|c|c|c|c|c|c|}
\hline Case & Total cost & Percent deviation & Fill rate(\%) & Avg. no. of $\mathrm{CO}-1$ & Avg. no. of $\mathrm{CO}-2$ \\
\hline $\mathrm{C} 1$ & 922.05 & 14.02 & 99.87 & 0.91 & 5.33 \\
\hline $\mathrm{C} 2$ & 1097.98 & 27.06 & 99.94 & 0.40 & 0.29 \\
\hline $\mathrm{C} 3$ & 1242.82 & 42.83 & 99.94 & 0.39 & 0.43 \\
\hline C4 & 1408.87 & 10.41 & 99.83 & 0.95 & 6.89 \\
\hline $\mathrm{C} 5$ & 1561.92 & 17.24 & 99.94 & 0.37 & 0.80 \\
\hline C6 & 1711.99 & 27.09 & 99.94 & 0.35 & 0.27 \\
\hline $\mathrm{C7}$ & 2445.98 & 10.29 & 99.71 & 1.31 & 12.08 \\
\hline $\mathrm{C} 8$ & 2518.47 & 10.56 & 99.93 & 0.35 & 0.24 \\
\hline C9 & 2522.69 & 10.59 & 99.97 & 0.32 & 0.36 \\
\hline
\end{tabular}

Number of items demanded at each arrival has a mean of 6 and a variance of 2/3. 
Table 11

Detailed results for selected compound Poisson cases with $10 \%$ error level.

\begin{tabular}{|c|c|c|c|c|c|}
\hline Case & Total cost & Percent deviation & Fill rate(\%) & Avg. no. of $\mathrm{CO}-1$ & Avg. no. of $\mathrm{CO}-2$ \\
\hline C11 & 933.78 & 13.92 & 99.79 & 1.06 & 6.37 \\
\hline C12 & 1179.09 & 33.44 & 99.91 & 0.42 & 0.49 \\
\hline $\mathrm{C} 13$ & 1300.66 & 45.48 & 99.93 & 0.40 & 0.42 \\
\hline C14 & 1425.08 & 10.36 & 99.80 & 0.98 & 6.76 \\
\hline C15 & 1643.95 & 21.75 & 99.91 & 0.46 & 0.91 \\
\hline $\mathrm{C} 16$ & 1850.09 & 35.12 & 99.91 & 0.44 & 0.48 \\
\hline $\mathrm{C} 17$ & 2399.49 & 8.09 & 99.76 & 0.99 & 8.22 \\
\hline C18 & 2663.80 & 15.72 & 99.88 & 0.53 & 2.51 \\
\hline C19 & 2775.59 & 19.26 & 99.91 & 0.42 & 0.58 \\
\hline
\end{tabular}

Number of items demanded at each arrival has a mean of 6 and a variance of 8 .

Property 4. As $r$ increases, the value of $Q^{*}$ decreases. This is a property that holds for regular inventory systems as well.

Property 5. $Q^{*}$ is a decreasing function of $p$. In other words, the derivative $d Q^{*} / d p<0$. This means that, if we have the opportunity of specifying an optimal value for $Q$, namely $Q^{*}$, this value will decrease as the error level increases.

Property 6. For constant $K_{q}$, the optimal value of $Q^{*}$ is an increasing function of $q$.

\section{Appendix B}

The following tables provide detailed results from selected simulation experiments. Tables 8 and 9 present data on simulations with unit Poisson demand at error levels of $1 \%, 5 \%$, $10 \%$ and $20 \%$. Tables 10 and 11 present data on simulations with compound Poisson demand at 10\% error level. Tables 10 and 11 are different with respect to the assumption on the distribution of demand at each demand arrival; both have the same means but the first set of cases have a demand variance of $2 / 3$ whereas the second set of cases have a demand variance of 8 .

\section{References}

Atalı, A., Lee, H., and Özer, O. 2009, If the inventory manager knew-value of visibility and RFID under imperfect inventory information. Technical report. Available at SSRN: 〈http://ssrn.com/abstract=1351606 >.

Beck, A. and Chapman, P. 2003, Hot spots in the supply chain: Developing and understanding of what makes some retail stores vulnerable to shrinkage. ECR Europe document available at the web site: <http://www.kamcity.com/ library/articles/hotspots.htm >.

Bensoussan, A., Sethi, C.M.S., 2005. Partially observed inventory systems: the case of zero-balance walk. International Journal of Production Economics 95 (1), 373-385.

Bernard, P.A., 1985. Cycle counting: the missing link. Production and Inventory Management 25 (4), 27-40.

Buck, J.R., Sadowski, R.P., 1983. Optimum stratified sampling. IIE Transactions 15, 119-126.

Cantwell, J., 1985. The how and why of cycle counting: the ABC method. Production and Inventory Management Journal 26 (2), 50-55.

DeHoratius, N., Mersereau, A.J., Schrage, L., 2008. Retail inventory management when records are inaccurate. Manufacturing and Service Operations Management 10 (2), 257-277.

Edelman, M.P., 1984. Cycle counting for distribution inventories. Readings in Production and Inventory Control and Planning APICS 29.
Fleisch, E., Tellkamp, C., 2005. Inventory inaccuracy and supply chain performance: a simulation study of a retail supply chain. International Journal of Production Economics 95 (1), 373-385.

Graff, R.T., 1987. The limitations of cycle counting. Production and Inventory Management Journal 28 (4), 39-43.

Gümrükçü, S., Rossetti, M.D., Buyurgan, N., 2008. Quantifying the cost of cycle counting in a two-echelon supply chain with multiple items. Internationa Journal of Production Economics 116, 263-274.

Hopp, W.J., Spearman, M.L., 1996. Factory Physics: Foundations of Manufacturing Management. McGraw-Hill, Burr Ridge, IL.

Iglehart, D.L., Morey, R.C., 1972. Inventory systems with imperfect asset information. Management Science 18 (8), 388-394.

Kang, Y., Gershwin, S.B., 2005. Information inaccuracy in inventory systems: Stock loss and stockout. IIE Transactions 37, 843-859.

Kok, A.G., Shang, K.H., 2007. Inspection and replenishment policies for system with inventory record inaccuracy. Manufacturing and Service Operations Management 9 (2), 185-205.

Lee, H.L., Özer, O., 2007. Unlocking the value of RFID. Production and Operations Management 16 (1), 40-64.

Martin, J.W., Goodrich, P.S., 1987. Minimizing sample size for given accuracy in cycle counting. Production and Inventory Management Journal 28 (4), 24-27.

Morey, R.C., 1985. Estimating service level impacts from changes in cycle count, buffer stock, or corrective action. Journal of Operations Management 5 (4), 411-418.

Morey, R.C., Dittman, D.A., 1986. Optimal timing of account audits in internal control. Management Science 32 (3), 272-282.

Mosconi, R., Raman, A., Zotteri, G., 2004. The impact of data quality and zerobalance walks on retail inventory management. Technical Report, Harvard Business School, Cambridge, MA.

Neely, P., 1987. Simple mathematics applied to inventory accuracy. Production and Inventory Management 28 (3), 64-68.

Raman, A., DeHoratius, N., Ton, Z., 2001. Execution the missing link in retai operations. California Management Review 43, 136-152.

Reddock, J.G., 1984. How to count a cycle. Readings in Production and Inventory Control and Planning APICS 28.

Rekik, Y., Jemai, Z., Sahin, E., Dallery, Y., 2007. Improving the performance of retail stores subject to execution errors: coordination versus RFID technology. OR Spectrum 29, 207-233.

Rekik, Y., Sahin, E., Dallery, Y., 2008a. Analysis of the impact of the RFID technology on reducing product misplacement errors at retail stores. International Journa of Production Economics 112, 264-278.

Rekik, Y., Sahin, E., Jemai, Z., Dallery, Y., 2008b. Execution errors in retail supply chains: analysis of the case of misplaced products. International Journal of Systems Science 39 (7), 727-740.

Rekik, Y., Sahin, E., Dallery, Y., 2009. Inventory accuracy in retail stores due to theft: an analysis of the benefits of RFID. International Journal of Production Economics 118, 189-198.

Sahin, E., Dallery, Y., 2009. Assessing the impact of inventory inaccuracies within a newsvendor framework. European Journal of Operational Research 197 1108-1118.

Sahin, E., Buzacott, J., Dallery, Y., 2008. Analysis of a newsvendor which has errors in inventory data records. European Journal of Operational Research 188, 370-389.

Young, S.T., Nie, W.D., 1992. A cycle-count model considering inventory policy and record variance. Production and Inventory Management Journal 33 (1), 11-17. 